\title{
Vascular normalization therapy with targeted localized vessel bevacizumab infusion in hepatocellular carcinoma after transarterial chemoembolization failure
}

\author{
Haixiao Zhang", Gengfei Cao", Weixin Ren, Junpeng Gu, Weizheng Ji, Diwen Zhu, Yingjun Bao, \\ Asihaer Hasimu \\ Interventional Radiology Department, The First Affiliated Hospital of Xinjiang Medical University, Wulumuqi, China \\ Contributions: (I) Conception and design: W Ji; (II) Administrative support: J Gu; (III) Provision of study materials or patients: H Zhang, G Cao, D \\ Zhu; (IV) Collection and assembly of data: Y Bao; (V) Data analysis and interpretation: A Hasimu; (VI) Manuscript writing: All authors; (VII) Final \\ approval of manuscript: All authors. \\ \#These authors contributed equally to this work. \\ Correspondence to: Weixin Ren. Interventional Radiology Department, The First Affiliated Hospital of Xinjiang Medical University, No. 137 Liyushan \\ Road, Wulumuqi 830054, China. Email: rwx1031@163.com.
}

\begin{abstract}
Background Hepatocellular carcinoma (HCC) is a highly vascularized tumor in which abnormal blood vessels contribute to poor treatment efficacy and prognosis. In this study, we assessed the efficacy, safety, and potential ability of bevacizumab to normalize tumor vascularity in patients with advanced HCC.

Methods: Patients with histologically or clinically confirmed advanced HCC that were refractory to conventional transarterial chemoembolization (c-TACE) received a transarterial infusion of bevacizumab ( $5 \mathrm{mg} / \mathrm{kg}$ ), followed by c-TACE (named as BEVA-TACE). The primary endpoint was overall survival (OS), which was defined as the time from a patient identified as TACE refractory to the occurrence of death. The secondary endpoints included progression-free survival (PFS) and the disease control rate (DCR).

Results: From January 2014 to December 2017, 20 patients with Barcelona Clinic Liver Cancer (BCLC) staging scores C $(80.0 \%)$ or D $(20.0 \%)$ received BEVA-TACE. The median OS time was 9.2 months [95\% confidence interval (CI): 2.1-22.6 months]. The median PFS time was 6.3 months (95\% CI: $1.0-10.5$ months). Despite the late stage, 1 patient (5.0\%) had a complete response (CR), 6 patients $(30.0 \%)$ had a partial response (PR), and 10 patients $(50.0 \%)$ had stable disease (SD) [overall response rate (ORR) 30.0\%; DCR 85.0\%]. The most common adverse events (AEs) were postembolic syndrome (25\%), hyperbilirubinemia $(10.0 \%)$, and melena $(10.0 \%)$. Severe III-IV oral mucositis and hypertension were observed in only 1 patient $(5.0 \%)$ during the follow-up period.
\end{abstract}

Conclusions: BEVA-TACE showed clinical efficacy, and patients with TACE-refractory HCC had acceptable AE rates. A low dose of targeted localized vessel bevacizumab infusion may normalize the condition of tumor blood vessels in patients with advanced HCC.

Keywords! Hepatocellular carcinoma (HCC); bevacizumab; vascular normalization therapy

Submitted Jul 07, 2021. Accepted for publication Aug 19, 2021.

doi: 10.21037/apm-21-2123

View this article at: https://dx.doi.org/10.21037/apm-21-2123

\section{Introduction}

Unresectable hepatocellular carcinoma (HCC) is a refractory disease that occurs after the failure of first- or second-line transarterial chemoembolization (TACE) and targeted therapy with sorafenib (1-3). Despite intense research efforts, no consensus has been reached concerning the management of refractory TACE or TACE failure.

Bevacizumab is a recombinant humanized monoclonal 
antibody (mAb) that blocks angiogenesis by inhibiting vascular endothelial growth factor A (VEGF-A). In combination with other chemotherapeutic drugs or immune checkpoint inhibitors, bevacizumab has been approved for the treatment of colorectal cancer (CRC), non-small cell lung cancer, metastatic breast cancer, advanced kidney cancer, and more recently, advanced HCC. Bevacizumab is recommended as a first-line therapy for advanced CRC (4), but the efficacy of its infusion through the hepatic artery has not been confirmed in HCC. In one study, intraarterial bevacizumab treatment was used in patients with recurrent glioblastoma and CRC liver metastasis, and the results showed that it was more cost-effective and had greater therapeutic effect than did conventional intravenous injection $(5,6)$.

Inspired by the success of the intra-arterial delivery approach for bevacizumab in the treatment of recurrent glioblastoma and CRC, we conducted a clinical trial (identifier No. ChiCTR-OPC-15006859) to evaluate the effectiveness of a low dose of targeted localized vessel bevacizumab infusion for advanced HCC patients resistant to TACE. We also assessed the antitumor activity and safety of bevacizumab infused directly into tumor vessels in patients with advanced HCC. We present the following article in accordance with the TREND reporting checklist (available at https://dx.doi.org/10.21037/apm-21-2123).

\section{Methods}

\section{Patients}

Patients were eligible for enrollment in the study if they had histologically or clinically confirmed advanced HCC that was not suitable for radical treatment (e.g., liver transplantation, resection, percutaneous ablation). Patients eligible to participate in the study must meet the following main inclusion criteria: (I) have HCC refractory to conventional TACE (c-TACE); (II) have a measurable disease according to the Response Evaluation Criteria in Solid Tumors (RECIST, version 1.0); (III) be 18 years of age or older; (IV) have an Eastern Cooperative Oncology Group performance status (ECOG-PS) score of 0-2; (V) have Child-Pugh class A/B/C; (VI) have no encephalopathy or ascites or need pleural effusion treatment; (VII) have a life expectancy $>12$ weeks; (VIII) have adequate hepatic and renal function as indicated by serum albumin $\geq 28 \mathrm{~g} / \mathrm{L}$ and total bilirubin $<2 \mathrm{mg} / \mathrm{dL}$, respectively; (IX) have alanine transaminase (ALT) and aspartate transaminase (AST) levels $<5$ times the normal value; $(\mathrm{X})$ have blood urea nitrogen and serum creatinine levels $<1.5$ times the normal value; (XI) have an international normalized ratio (INR) $<1.7$; and (XII) have a platelet count $\geq 50 \times 10^{9} / \mathrm{L}$. All patients provided written informed consent during the treatment phase.

Patients were excluded from the study if they met any of the following exclusion criteria: had a biliary obstruction due to tumor invasion, a definite hepatic artery-portal vein fistula, extensive portal vein cancer embolus with Child-Pugh C, clinically apparent central nervous system metastases or carcinomatous meningitis, uncontrolled hypertension, a history of hemorrhage of the digestive tract within 4 weeks, a history of a major operation within 4 weeks, a combination of other malignancies, and/or history of any antiangiogenesis therapy within 4 weeks.

In this study, a patient was classified as having TACErefractory status if, after at least 2 consecutive TACE procedures, the tumor still progressed, or a new lesion, vascular invasion, or extra-hepatic metastases appeared.

The trial was registered in Chinese clinical trial registry (https://www.chictr.org.cn), ID: ChiCTR-OPC-15006859. The study protocol was approved by the Ethics Committee of The First Affiliated Hospital of Xinjiang Medical University (Urumqi, Xinjiang, China; approved No. of Ethic Committee: 20150507-07). All the procedures were performed in accordance with the ethical standards of the responsible committee on human experimentation of The First Affiliated Hospital of Xinjiang Medical University and in compliance with the Helsinki Declaration (as revised in 2013). The need for written informed consent was waived in the stages of patient data collection, statistical analysis, and publication due to the retrospective nature of the study. Data of patients' disease history, physical examination, serum laboratory tests, and radiologic investigations [computed tomography (CT) or magnetic resonance imaging (MRI)] were collected from patients' hospital records.

\section{Treatment protocol}

All patients received a transarterial infusion of bevacizumab, followed by c-TACE (BEVA-TACE). After a microcatheter was inserted into the tumor-feeding artery, bevacizumab $(5 \mathrm{mg} / \mathrm{kg})$ was administered as a 30 -minute transcatheter infusion. A standard c-TACE was then conducted using lipiodol and embolic microspheres or a gelatin sponge. 


\section{Follow-up period}

All patients were followed up every 4-6 weeks (starting immediately after the treatment) by laboratory blood samples examination and contrast-enhanced CT or MRI.

\section{Study assessments}

The primary outcome was overall survival (OS), which was defined as the time from a patient identified as TACE refractory to the occurrence of death due to any cause or until the last follow-up appointment. The secondary outcomes were progression-free survival (PFS) and disease control rate (DCR). PFS was defined as the time taken to identify patients as TACE refractory until radiologists diagnose tumor progression according to the modified RECIST (mRECIST) criteria. In relation patients who died or patients lost during the follow-up period, the date at which the last time radiological assessment was conducted was considered the withdrawal date. The DCR was defined as the proportion of patients with advanced HCC who obtained either a complete response (CR), partial response (PR), or progression-free stable disease (SD) aftertreatment.

Tumor response was evaluated according to the mRECIST criteria based on enhanced CT or MRI results collected during the follow-up visits. CR was defined as the disappearance of the tumor enhancement area during the arterial phase, reflecting the complete necrosis of the tissue. $\mathrm{PR}$ was defined as the reduction of tumor area by more than $30 \%$, and progressive disease (PD) was considered to have increased by at least 20\% (of the sum of the longest diameter) in the enhanced tumor areas. SD was defined as either sufficient shrinkage (qualified as PR) or a sufficient increase in the tumor (qualified as PD).

\section{Safety monitoring}

The safety assessments included monitoring and recording all adverse events (AEs) and serious AEs throughout the study period. In addition to the monitoring of vital signs, the urine, hematology, and blood chemistry data of the enrolled patients were regularly collected.

\section{Statistical analysis}

Statistical analyses were performed using $\mathrm{R}$ version 3.3.1 (The R Foundation for Statistical Computing) for Windows. For the characteristics of the participants continuous variables are expressed as mean and standard deviation, while categorical variables are expressed as frequency and percentage. The categorical variables were compared using $\chi^{2}$ test or Fisher's exact test. The continuous variables were compared using a $t$-test or Mann-Whitney $\mathrm{U}$ test depending on the data distribution. The survival curves were estimated using the Kaplan-Meier method. A twotailed $\mathrm{P}$ value $<0.05$ was considered to a indicate statistically significant difference in all the analyses.

\section{Results}

\section{Patient characteristics}

From January 2014 to December 2017, 20 patients with histologically or clinically confirmed advanced HCC who were refractory to c-TACE were enrolled in this study. The overall staging was relatively late (see Table 1); 16 (80.0\%) patients had Barcelona Clinic Liver Cancer (BCLC) staging score $\mathrm{C}$, and the other $4(20 \%)$ patients had staging score D; the ECOG-PS score of 15 (75.0\%) patients was 2, while that of the remaining 5 patients $(25.0 \%)$ was 3 ; only $6(30.0 \%)$ patients had the opportunity to receive surgery before enrollment in the study; $3(15.0 \%)$ patients accepted oral sorafenib treatment before enrollment in the study, and 8 (40.0\%) patients received other systemic chemotherapy. On average, the patients underwent TACE treatment 3.4 times.

\section{Efficacy}

All enrolled patients underwent an average of 1.6 [1-3] sessions of BEVA-TACE. Patients' had a median OS time of 9.2 months [95\% confidence interval (CI) 2.1-22.6 months; see Figure $1 A$ ] and a median PFS time of 6.3 months $(95 \%$ CI 1.0-10.5 months; see Figure 1B). Tumor reduction of the target lesions, which was assessed according to the mRECIST criteria based on dynamic CT or MRI scans, occurred in 12 (60.0\%) patients. Of all 20 late-staging HCC patients, 1 patient $(5.0 \%)$ showed CR, 6 patients showed PR (30.0\%), 10 patients (50.0\%) showed SD, and 3 patients (15.0\%) showed PD [overall response rate (ORR): $30.0 \%$; DCR: $85.0 \%$; see Figure 2]. As Figure 3 shows, transarterial infusion of bevacizumab significantly mitigated hepatic vascular permeability and interstitial edema, which were important manifestations of vascular normalization.

\section{Safety}

All 20 patients experienced at least $1 \mathrm{AE}$. The most common any-grade AEs (see Table 2) were elevated transaminase 
Table 1 Patient demographics and baseline characteristics

\begin{tabular}{|c|c|}
\hline Characteristics & Patient $(n=20)$ \\
\hline Median age, years [range] & $54.5[35-78]$ \\
\hline \multicolumn{2}{|l|}{ Sex, n (\%) } \\
\hline Female & $2(10.0)$ \\
\hline Male & $18(90.0)$ \\
\hline \multicolumn{2}{|l|}{ ECOG-PS, n (\%) } \\
\hline 2 & $15(75.0)$ \\
\hline 3 & $5(25.0)$ \\
\hline \multicolumn{2}{|l|}{ Child-Pugh class, n (\%) } \\
\hline$A$ & $3(15.0)$ \\
\hline$B$ & $16(80.0)$ \\
\hline C & $1(5.0)$ \\
\hline \multicolumn{2}{|l|}{ BCLC staging, $\mathrm{n}(\%)$} \\
\hline C & $16(80.0)$ \\
\hline $\mathrm{D}$ & $4(20.0)$ \\
\hline \multicolumn{2}{|l|}{ Cause of $\mathrm{HCC}, \mathrm{n}(\%)$} \\
\hline Hepatitis B & $16(80.0)$ \\
\hline Alcohol & $1(5.0)$ \\
\hline Alcohol and hepatitis B & $1(5.0)$ \\
\hline Unknown & $2(10.0)$ \\
\hline \multicolumn{2}{|l|}{ AFP value at baseline, $n(\%)$} \\
\hline$<200 \mathrm{ng} / \mathrm{mL}$ & $5(25.0)$ \\
\hline$\geq 200 \mathrm{ng} / \mathrm{mL}$ & $15(75.0)$ \\
\hline Times of TACE [range] & $3.5[2-9]$ \\
\hline \multicolumn{2}{|l|}{ Prior surgery for $\mathrm{HCC}, \mathrm{n}(\%)$} \\
\hline No & $14(70.0)$ \\
\hline Yes & $6(30.0)$ \\
\hline \multicolumn{2}{|l|}{ Prior RFA therapy, n (\%) } \\
\hline No & $14(70.0)$ \\
\hline Yes & $6(30.0)$ \\
\hline \multicolumn{2}{|l|}{ Prior chemotherapy, n (\%) } \\
\hline Sorafenib & $3(15.0)$ \\
\hline Other systemic chemotherapy & $8(40.0)$ \\
\hline
\end{tabular}

ECOG-PS, Eastern Cooperative Oncology Group performance status; BCLC, Barcelona Clinic Liver Cancer; HCC, hepatocellular carcinoma; AFP, alpha-fetoprotein; TACE, transarterial chemoembolization; RFA, radiofrequency ablation.
(16, 80\%), hyperbilirubinemia $(9,45 \%)$ and nausea (7, 35\%). A further 2 patients (10\%) had anaphylaxis and 2 $(10 \%)$ had melena, which is considered to be associated with bevacizumab treatment. No treatment-related deaths were reported. AEs were generally manageable with symptomatic treatment.

\section{Discussion}

TACE, which combines chemotherapy and the occlusion of the tumor blood supply, remains the standard treatment for patients with intermediate and advanced HCC (7-9). However, the treated tumors revascularize and require retreatment after an initial objective tumoral response in $25-40 \%$ of patients. This conventional treatment aims to deprive the tumor of oxygen and nutrients, and has been associated with increases in metastasis and decreases in the delivery of chemo-, radio-, and immune therapies (10). This creates a feedback loop, in which repeated vessel embolization increases new angiogenesis.

Blood supply normalization in HCC treatment, which leads to increased tumor perfusion and oxygenation, is an emerging concept. A growing number of preclinical and clinical studies of vessel normalization agents have documented their antitumor activity against HCC (11-14). An earlier landmark study revealed that a single dose of bevacizumab in rectal carcinoma patients reduced microvascular density and improved vessel permeability, pericyte coverage, and interstitial hypertension $(15,16)$. However, it is important to recognize that sustained or high doses of bevacizumab may result in the excessive regression of the vasculature, creating a hypoxic microenvironment, while low doses may cause tumoral vascular normalization; this delicate balance has been termed the "normalization window" $(10,17)$. In our study, the dose of bevacizumab in the transarterial infusion $(5 \mathrm{mg} / \mathrm{kg}$ ) was less than that of the conventional intravenous injection $(7.5$ or $15 \mathrm{mg} / \mathrm{kg}$ ). Indeed, a low dose is key to achieving vascular normalization as opposed to antiangiogenesis.

In relation to HCC-directed vascular normalization therapies, it is important to note that in addition to the effects on vessel normalization of bevacizumab and other agents, including thalidomide, sorafenib, sunitinib, lenvatinib, and regorafenib, being unknown, the normalization window is still debatable.

We speculated that a low dose of targeted vessel localized bevacizumab infusion could normalize the tumor blood vessels 

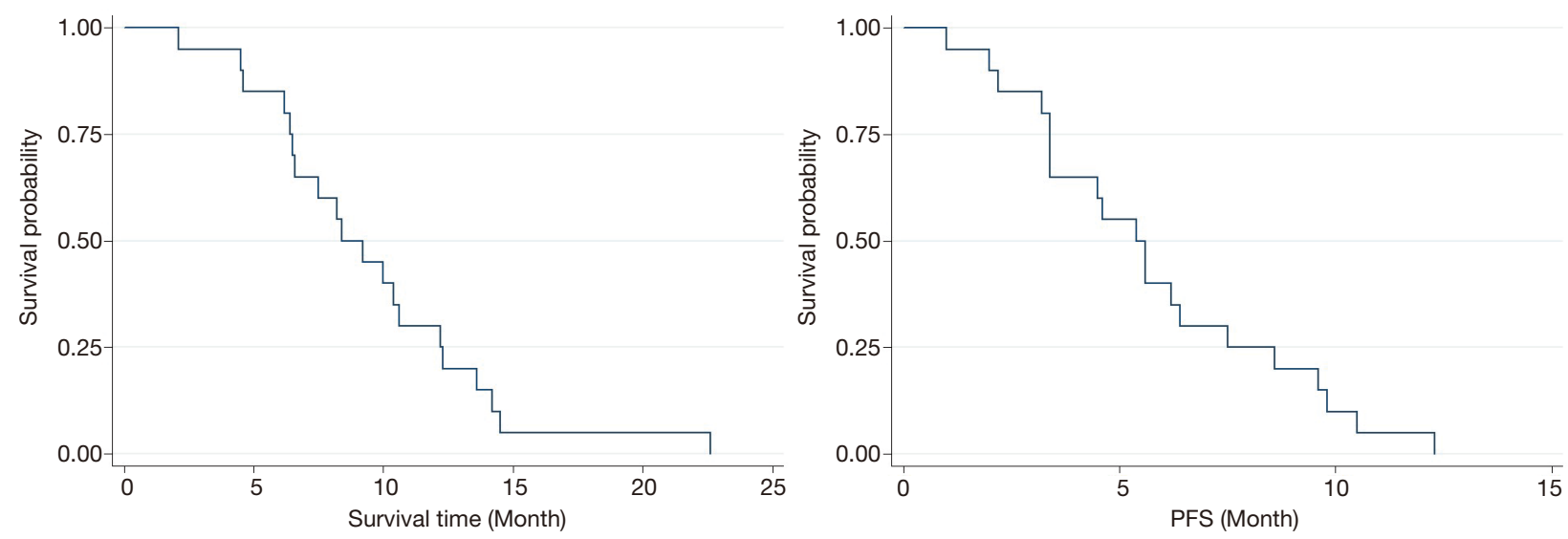

Figure 1 Kaplan-Meier estimates of OS and PFS. (A) The median OS was 9.2 months (95\% CI: 2.1-22.6 months). (B) The median PFS was 6.3 months (95\% CI: 1.0-10.5 months). OS, overall survival; PFS, progression-free survival; CI, confidence interval.

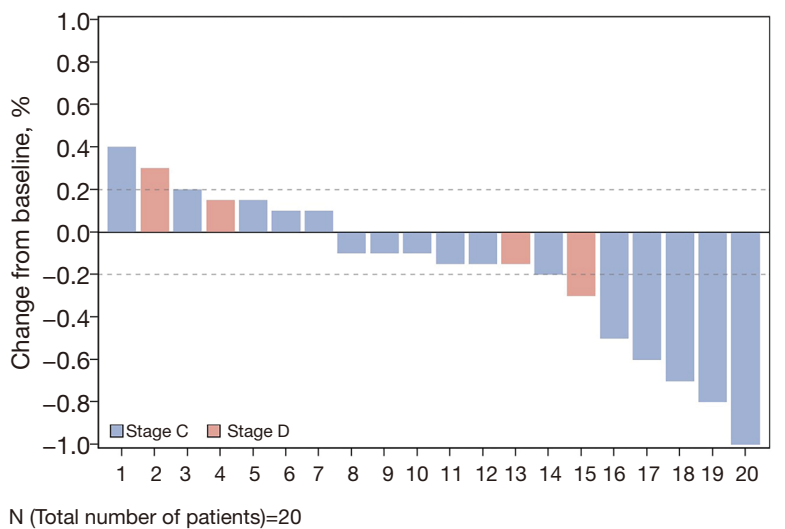

Figure 2 Waterfall plot of changes in tumor size according to the mRECIST criteria. mRECIST, modified Response Evaluation Criteria in Solid Tumors.

in patients with TACE-refractory HCC. This approach is the opposite of the traditional method adopted at our center in which the blood supply of the tumor is deprived to improve the OS and PFS of patients with TACE-resistant HCC.

We confirmed that severe abdominal pain due to hepatic enlargement in some patients was totally relieved within 24 hours. The manifestations of vascular normalization, such as a reduced vessel diameter and tortuosity, and a decrease in vascular permeability and interstitial edema were revealed by digital subtraction angiography and CT scan 30 days after the initial therapy. When implementing treatment with transarterial bevacizumab infusion for HCC, we recommend the following: (I) accurate preoperative evaluation regarding refractory TACE or TACE failure. Indeed, all the patients enrolled in our study either experience refractory TACE or TACE failure. The indications for this treatment should not be expanded until there is new evidence. (II) The dosage of bevacizumab should be strictly controlled. As mentioned above, a low dose of bevacizumab is the key to achieving vascular normalization and can also reduce the economic burden of patients. Therefore, the dosage should be controlled at $5 \mathrm{mg} / \mathrm{kg}$. (III) Transarterial infusion of bevacizumab is the key step during treatment, but embolization with lipiodol is also critical. The association of embolization with bevacizumab creates a synergistic interaction.

There were several limitations to this study. First, this was a single center retrospective cohort study, which is prone to various deviations, including the lack of control group and possible confusion caused by unmeasured variables Second, due to the nature of the experimental design, the sample size in our study was relatively small, which might have reduced the statistical power. The enrolled patients had no standard treatment at the time of enrollment, and, as an exploratory treatment measure, we did not try to expand the number of patients at the beginning, as we adopted a cautious approach concerning the safety and efficacy of the treatment.

In conclusion, targeted localized vessel bevacizumab infusion showed clinical activity and acceptable toxicity profiles in patients with TACE-refractory HCC. We suggest that when no other effective treatments are available, this treatment be used as an alternative treatment. 

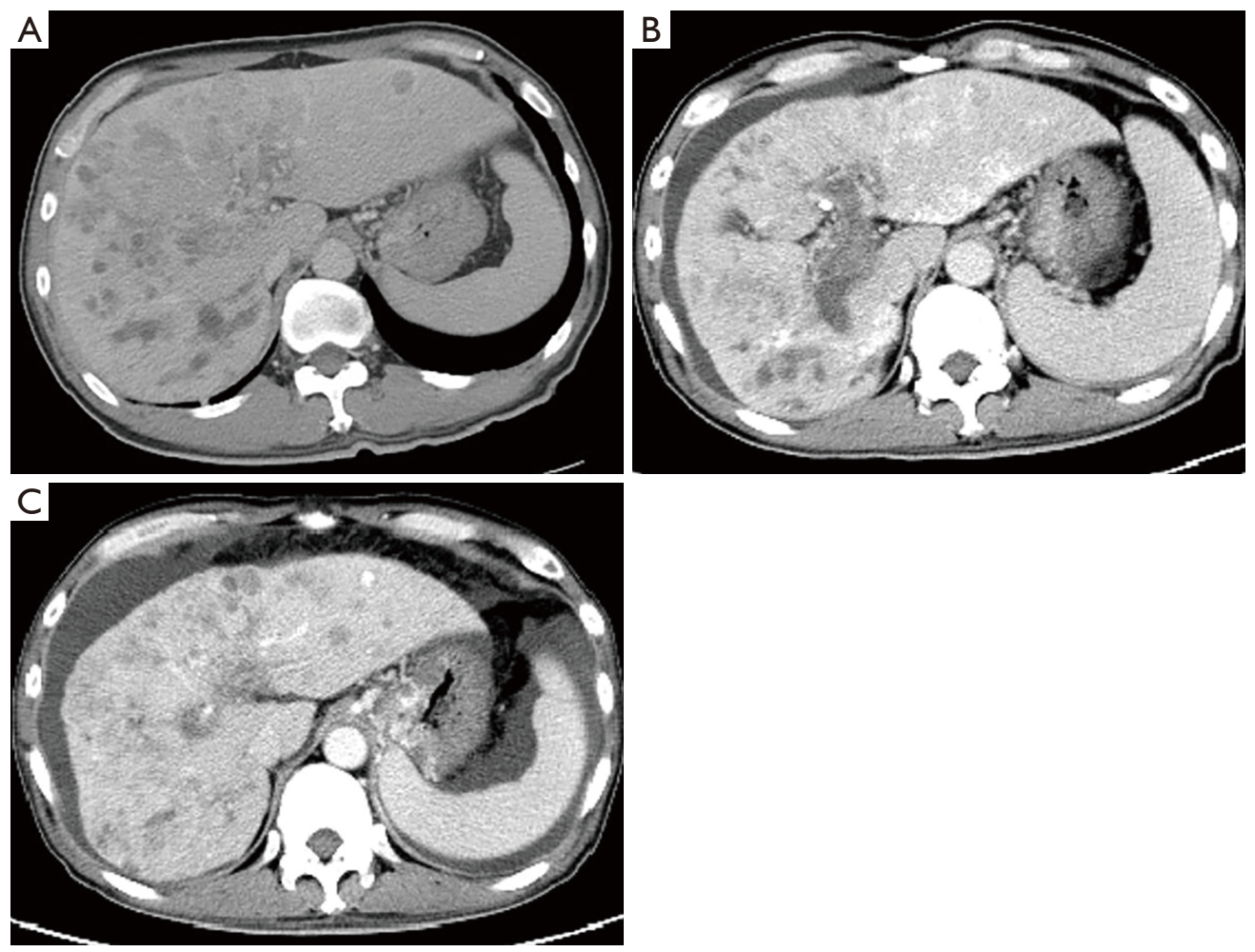

Figure 3 A 52-year-old male patient had right upper abdominal pain and fatigue for 2 months. Physical examination showed hardened liver tissues $5 \mathrm{~cm}$ below the xiphoid. ECOG-PS score 3; liver function: Child-Pugh B; AFP >1,000 ng/mL. The symptom of right upper abdominal pain was relieved 24 hours after BEVA-TACE, while fatigue was relieved 1 week after the treatment. The ECOG score was improved from 3 to 1. (A) A CT scan showing hepatomegaly with multiple lesions across the right and left lobe. (B) Liver enlargement was significantly decreased in less than 2 months after bevacirumab treatment. (C) A CT scan 6 months after BEVA-TACE showing that the volume of the liver was continuing to shrink. BEVA-TACE had greatly mitigated hepatic vascular permeability and interstitial edema, which were the important manifestations of vascular normalization. The patient had a PFS of 9 months and a survival period 13 months. ECOG-PS, Eastern Cooperative Oncology Group performance status; AFP, alpha-fetoprotein; CT, computed tomography; BEVA-TACE, bevacizumab $(5 \mathrm{mg} / \mathrm{kg})$, followed by conventional transarterial chemoembolization; PFS, progression-free survival.

Table 2 Common AEs occurring in all the patients

\begin{tabular}{|c|c|c|c|c|c|}
\hline$A E$ & Any grade, $n$ [\%] & \multicolumn{4}{|c|}{ Grade (n) } \\
\hline Elevated transaminase & 16 [80] & 12 & 4 & - & - \\
\hline Hyperbilirubinemia & $9[45]$ & 7 & 2 & - & - \\
\hline Nausea & $7[35]$ & 7 & - & - & - \\
\hline Fever & 3 [15] & 2 & 1 & - & - \\
\hline Anaphylaxis & $2[10]$ & 2 & - & - & - \\
\hline Melena & $2[10]$ & 2 & - & - & - \\
\hline Hypertension & $1[5]$ & 0 & 1 & - & - \\
\hline
\end{tabular}

AE, adverse event. 


\section{Acknowledgments}

Funding: None.

\section{Footnote}

Reporting Checklist: The authors have completed the TREND reporting checklist. Available at https://dx.doi. org/10.21037/apm-21-2123

Data Sharing Statement: Available at https://dx.doi. org/10.21037/apm-21-2123

Conflicts of Interest: All authors have completed the ICMJE uniform disclosure form (available at https://dx.doi. org/10.21037/apm-21-2123). The authors have no conflicts of interest to declare.

Ethical Statement: The authors are accountable for all aspects of the work in ensuring that questions related to the accuracy or integrity of any part of the work are appropriately investigated and resolved. The study protocol was approved by the Ethics Committee of The First Affiliated Hospital of Xinjiang Medical University (Urumqi, Xinjiang, China; approved No. of Ethic Committee: 20150507-07). All the procedures were performed in accordance with the ethical standards of the responsible committee on human experimentation of The First Affiliated Hospital of Xinjiang Medical University and in compliance with the Helsinki Declaration (as revised in 2013). The need for written informed consent was waived in the stages of patient data collection, statistical analysis, and publication due to the retrospective nature of the study.

Open Access Statement: This is an Open Access article distributed in accordance with the Creative Commons Attribution-NonCommercial-NoDerivs 4.0 International License (CC BY-NC-ND 4.0), which permits the noncommercial replication and distribution of the article with the strict proviso that no changes or edits are made and the original work is properly cited (including links to both the formal publication through the relevant DOI and the license). See: https://creativecommons.org/licenses/by-nc-nd/4.0/.

\section{References}

1. Kudo M, Han KH, Ye SL, et al. A changing paradigm for the treatment of intermediate-stage hepatocellular carcinoma: Asia-Pacific Primary Liver Cancer Expert Consensus Statements. Liver Cancer 2020;9:245-60.

2. Ogasawara S, Ooka Y, Koroki K, et al. Switching to systemic therapy after locoregional treatment failure: definition and best timing. Clin Mol Hepatol 2020;26:155-62.

3. Wu J, Li A, Yang J, et al. Efficacy and safety of TACE in combination with sorafenib for the treatment of TACErefractory advanced hepatocellular carcinoma in Chinese patients: a retrospective study. Onco Targets Ther 2017;10:2761-8.

4. Modest DP, Pant S, Sartore-Bianchi A. Treatment sequencing in metastatic colorectal cancer. Eur J Cancer 2019;109:70-83.

5. Galla N, Chiang G, Chakraborty S, et al. Apparent diffusion coefficient changes predict survival after intraarterial bevacizumab treatment in recurrent glioblastoma. Neuroradiology 2017;59:499-505.

6. Chen H, Zhang J, Cao G, et al. Target hepatic artery regional chemotherapy and bevacizumab perfusion in liver metastatic colorectal cancer after failure of first-line or second-line systemic chemotherapy. Anticancer Drugs 2016;27:118-26.

7. Vogel A, Cervantes A, Chau I, et al. Hepatocellular carcinoma: ESMO Clinical Practice Guidelines for diagnosis, treatment and follow-up. Ann Oncol 2019;30:871-3. Erratum for: Ann Oncol 2018;29:iv238-iv55.

8. Heimbach JK, Kulik LM, Finn RS, et al. AASLD guidelines for the treatment of hepatocellular carcinoma. Hepatology 2018;67:358-80.

9. Omata M, Cheng AL, Kokudo N, et al. Asia-Pacific clinical practice guidelines on the management of hepatocellular carcinoma: a 2017 update. Hepatol Int 2017;11:317-70.

10. Jain RK. Normalizing tumor vasculature with antiangiogenic therapy: a new paradigm for combination therapy. Nat Med 2001;7:987-9.

11. Rolny C, Mazzone M, Tugues S, et al. HRG inhibits tumor growth and metastasis by inducing macrophage polarization and vessel normalization through downregulation of PlGF. Cancer Cell 2011;19:31-44.

12. Young JA, Ting KK, Li J, et al. Regulation of vascular leak and recovery from ischemic injury by general and VEcadherin-restricted miRNA antagonists of miR-27. Blood 2013;122:2911-9.

13. Zhang H, Ren Y, Tang X, et al. Vascular normalization induced by sinomenine hydrochloride results in suppressed mammary tumor growth and metastasis. Sci Rep $2015 ; 5: 8888$. 
14. Min L, Ling W, Hua R, et al. Anti-angiogenic therapy for normalization of tumor vasculature: a potential effect of Buyang Huanwu decoction on nude mice bearing human hepatocellular carcinoma xenografts with high metastatic potential. Mol Med Rep 2016;13:2518-26.

15. Willett CG, Boucher Y, di Tomaso E, et al. Direct evidence that the VEGF-specific antibody bevacizumab has antivascular effects in human rectal cancer. Nat Med 2004;10:145-7.

Cite this article as: Zhang $\mathrm{H}$, Cao G, Ren W, Gu J, Ji W, Zhu D, Bao Y, Hasimu A. Vascular normalization therapy with targeted localized vessel bevacizumab infusion in hepatocellular carcinoma after transarterial chemoembolization failure. Ann Palliat Med 2021;10(8):9149-9156. doi: 10.21037/apm-21-2123
16. Weisshardt P, Trarbach T, Dürig J, et al. Tumor vessel stabilization and remodeling by anti-angiogenic therapy with bevacizumab. Histochem Cell Biol 2012;137:391-401.

17. Winkler F, Kozin SV, Tong RT, et al. Kinetics of vascular normalization by VEGFR2 blockade governs brain tumor response to radiation: role of oxygenation, angiopoietin-1, and matrix metalloproteinases. Cancer Cell 2004;6:553-63.

(English Language Editor: L. Huleatt) 